Notre Dame Journal of Formal Logic

Volume 26, Number 2, April 1985

\title{
From Preference to Utility: A Problem of Descriptive Set Theory
}

\author{
JOHN P. BURGESS*
}

1 Introduction Some years ago J. H. Silver proved that a co-analytic equivalence relation on a Polish space has either countably many or continuum many equivalence classes. Later L. Harrington greatly simplified the complicated original proof. The present paper is a sort of footnote to Harrington's lectures on these matters. It will be shown that information developed in his proof settles a problem of (hyper-)theoretical mathematical economics first investigated by Wesley [13] and Mauldin [8]. Namely, it will be shown that any family of closed preference orders that is parametrized in a Borel fashion can be represented by a family of continuous utility functions parametrized in an absolutely measurable fashion. Though the author is greatly indebted to Mauldin's work [8], the treatment of the problem here will be self-contained. Background and motivation for problems of this kind can be found in [6], Section 2.1. Terminology and notation pertaining to descriptive set theory will be as in [9].

2 Definitions Throughout let $y$ be a topological space. A preference order on $q$ is any transitive, connected binary relation $\leqslant *$. Associated are the strict preference and indifference relations given by:

$$
\begin{aligned}
& x<^{*} y \longleftrightarrow x \leqslant^{*} y \& \sim y \leqslant^{*} x \\
& x \equiv * y \longleftrightarrow x \leqslant * y \& y \leqslant * x .
\end{aligned}
$$

Note that $\equiv^{*}$ is an equivalence relation, and that $\leqslant^{*}$ induces a linear order on its equivalence classes. $[x]^{*}$ will denote the equivalence class of $x . \leqslant^{*}$ will be

\footnotetext{
*Research in part supported by USA National Science Foundation Grant MCS 8003254.
} 
called closed if all sets of the forms $\{y: x \leqslant * y\}$ and $\{y: y \leqslant * x\}$ are closed; or in fancier language, if the given topology on $y$ is at least as fine as the order topology induced by $\leqslant^{*}$. When $y$ has a countable basis this condition implies (by Theorem 5 below) that $\leqslant^{*}$ is closed when considered as a subset of $\mathscr{Y} \times \mathscr{y}$. When $g$ is $T_{1}$, the converse implication holds. So our terminology is not entirely inappropriate.

A utility function on $y$ is any function $f$ taking values in the closed unit interval $[0,1]$. A utility function $f$ represents a preference order $\leqslant^{*}$ if we always have $x \leqslant * y$ iff $f(x) \leqslant f(y)$. Plainly a necessary condition for the existence of a representation by a continuous utility function $f$ is that $\leqslant^{*}$ should be closed; often this condition is also sufficient.

3 Proposition (cf. [8], Theorem 2.8) If oy is countable, then every preference order $\leqslant^{*}$ on $y$ can be represented by a utility function $\phi$; moreover, when $\leqslant^{*}$ is closed, $\phi$ can be taken as continuous.

Proof: Cantor's back-and-forth method yields a function $\Phi$ assigning each $\leqslant *$ a representation $\phi$ taking values in the rationals of the open unit interval, which moreover is "as near to surjective as possible" in the sense that we have $r \in(\mathbf{Q} \cap(0,1))$ - range $\phi$ only when one of the following holds:

$$
\begin{aligned}
& \exists x(\sim \exists z(x<* z) \& f(x)<r) \\
& \exists y(\sim \exists z(z<* y) \& r<f(y)) \\
& \exists x \exists y\left(x<<^{*} y \& \sim \exists z(x<* z<* y) \& f(x)<r<f(y)\right) .
\end{aligned}
$$

Plainly this condition implies continuity with respect to the order topology and any finer topology.

4 Supplement (cf. [8], Theorem 5.5) The set PO of all preference orders on $\omega$ is (when a relation is identified with its characteristic function) a closed subset of a homeomorph (namely $\omega \times \omega_{2}$ ) of the Cantor space $C$. The set $U F$ of all utility functions on $\omega$ is just (the underlying set of) the Hilbert cube ${ }^{\omega}[0,1]$. Thus both sets carry natural Polish topologies. Tedious but routine computations show that the function $\Phi$ of the foregoing proof is Borel-measurable with respect to these topologies.

5 Theorem (Debreu [3]; cf. [8] for a proof like that below and others) If of has a countable basis, then any closed preference order $\leqslant^{*}$ on $y$ can be represented by a continuous utility function $f$.

Proof: As a first try, fix a basis $\left(U_{i}: i \in \omega\right)$ and define:

$$
\begin{aligned}
& k_{i}(y)=\left\{\begin{array}{l}
1 \text { if } U_{i} \subseteq\left\{z: z \leqslant^{*} y\right\} \\
0 \text { otherwise }
\end{array}\right. \\
& k \quad=\frac{1}{2} \sum_{i} 2^{-i} k_{i} .
\end{aligned}
$$

It is readily verified that the utility function $k$ represents $\leqslant *$. Unfortunately, it may be only semicontinuous. But the existence of any representation at all, even a discontinuous one, has one important consequence: Call $[x]^{*}$ a jump class if:

$$
\exists y(x<* y \& \sim \exists z(x<* z<* y))
$$


Then the number of jump classes is countable (for the intervals $(k(x), k(y))$ arising from such classes are nonempty and disjoint).

As a second try, fix ${ }^{\#}$ a sequence $\left(z_{i}: i \epsilon \omega\right)$ dense in $y$, say by taking $z_{i} \in U_{i}$. Apply the function $\Phi$ of the proof of Proposition 3 to the restriction of $\leqslant^{*}$ to the $z_{i}$, obtaining a representation $\phi$. Extend this to a function $h$ on all of $\mathcal{y}$ by defining:

$$
h(y)=\left\{\begin{array}{l}
\inf \left\{\phi\left(z_{i}\right): y \leqslant z_{i}\right\} \text { if this set is nonempty } \\
1 \text { otherwise. }
\end{array}\right.
$$

It is not hard to see that the utility function $h$ is continuous (e.g., what is $h^{-1}[0, a)$ ? If there is no $z_{i} \leqslant^{*}$-maximal with respect to the property $\phi\left(z_{i}\right)<a$, then it is the union of the open sets $\left\{y: y<{ }^{*} z_{j}\right\}$ for those $z_{j}$ with $\phi\left(z_{j}\right)<a$. If there is such a $z_{i}$, then by "near surjectivity" there must also be a $z_{k} \leqslant^{*}$-minimal with respect to the property $\phi\left(z_{k}\right) \geqslant a$, and then the set in question is just $\left.\left\{y: y<^{*} z_{k}\right\}\right)$. Nor is it hard to see that we always have:

$$
x \leqslant * y \rightarrow h(x) \leqslant h(y) .
$$

Unfortunately, the converse may sometimes fail. But at least we can say:

$$
x<* y \& h(x)=h(y) \rightarrow[x]^{*} \text { is a jump class. }
$$

(For otherwise there must exist $z_{i}, z_{j}$ with $x<^{*} z_{i}<^{*} y$ and $x<^{*} z_{j}<^{*} z_{i}$, whence $h(x) \leqslant \phi\left(z_{j}\right)<\phi\left(z_{i}\right) \leqslant h(y)$. In fact, this shows that no more than three $\equiv *$-equivalence classes can be carried by $h$ to the same value $r$.)

As a third try, fix \#\# a sequence $\left(x_{i}: i \in \omega\right)$ containing at least one representative of each jump class. On account of the use we are eventually to make of this proof, we do not insist that each $\left[x_{i}\right]^{*}$ actually be a jump class, but rather we let $m_{i}$ be 1 or 0 according as it is or is not a jump class. Define:

$$
\begin{aligned}
f_{i}(y) & =\left\{\begin{array}{l}
1 \text { if } x_{i}<^{*} y \\
0 \text { otherwise }
\end{array}\right. \\
f^{\prime} & =\frac{1}{2} \sum_{i} m_{i} f_{i} \\
f & =\frac{1}{2} h+\frac{1}{2} f^{\prime} .
\end{aligned}
$$

It is readily verified that $f$ does everything required.

6 Alternative Proof: For future use, we offer a roundabout alternative to the above proof: $F i x^{\#}\left(z_{i}: i \in \omega\right)$ and define $h$ as above. Fix ${ }^{\# \# \#}$ a sequence $\left(r_{i}: i \in \omega\right)$ such that for all $r \in[0,1]$ we have:

$$
\exists x \exists y(x<* y \& h(x)=r=h(y)) \rightarrow \exists i r_{i}=r .
$$

Fix sequences $\left(x_{i}^{\prime}: i \in \omega\right),\left(x_{i}^{\prime \prime}: i \in \omega\right),\left(x_{i}^{\prime \prime \prime}: i \in \omega\right)$ such that:

$$
\begin{aligned}
& \exists x\left(h(x)=r_{i}\right) \rightarrow h\left(x_{i}^{\prime}\right)=r_{i} \\
& \exists x\left(x \not x^{*} x_{i}^{\prime} \& h(x)=r_{i}\right) \rightarrow x_{i}^{\prime \prime} \not x^{*} x_{i}^{\prime} \& h\left(x_{i}^{\prime \prime}\right)=r_{i} \\
& \exists x\left(x \not x^{*} x_{i}^{\prime} \& x \not \neq^{*} x_{i}^{\prime \prime} \& h(x)=r_{i}\right) \rightarrow x_{i}^{\prime \prime \prime} \not x^{*} x_{i}^{\prime} \& x_{i}^{\prime \prime \prime} \not x^{*} x_{i}^{\prime \prime} \& h\left(x_{i}^{\prime \prime \prime}\right)=r_{i} .
\end{aligned}
$$

Define a sequence $\left(x_{i}: i \in \omega\right)$ by letting $x_{3 i}=x_{i}^{\prime}, x_{3 i+1}=x_{i}^{\prime \prime}, x_{3 i+2}=x_{i}^{\prime \prime \prime}$. Then define $m_{i}, f_{i}, f^{\prime}$, and $f$ as above to complete the construction. 
7 Remark: Plainly the representation of Theorem 5 is not unique. Other representations can be obtained by composition with monotone continuous functions. In the proof, this nonuniqueness is reflected in the need for making arbitrary choices (at the places marked \# and \#\#). When we try to carry out the proof "parameter-wise" for an indexed family $\leqslant_{t}$ of preference orders, these choices will cause us trouble. To avoid appeal to the Axiom of Choice, we will have to invoke certain uniformization or selection theorems. (The roundabout Alternative Proof requires less powerful such theorems than the more direct original proof.) From the exhaustive catalogs [11], [12] we select and list below the uniformization theorems we will need. Logicians may prefer the proofs in [9], Part 4F, to those in the original papers.

8 Definitions Throughout let $\tilde{\sigma}, \mathcal{x}$ be Polish spaces. A function between such spaces is analytically measurable ( $C$-measurable, respectively) if it is measurable with respect to the smallest $\sigma$-field containing the analytic sets (containing the Borel sets and stable under operation $A$, respectively). Analytic measurability implies $C$-measurability which implies absolute measurability. The class of $C$-measurable functions is stable under composition as the class of analytically measurable functions is not.

9 Theorem (Yankov [14], von Neumann [10]) Let $A \subseteq \mathcal{J} \times \mathscr{\%}$ be analytic. Then $A$ can be uniformized by an analytically measurable function.

10 Theorem Let $B \subseteq \mathcal{J} \times$ x $_{0}$ be Borel with each fiber $B_{t}=\{x:(t, x) \in B\}$ $\sigma$-compact.

(a) (Arsenin [1]) The projection of $B$ to $\sigma$ is Borel.

(b) (Choban [2]) B can be uniformized by a Borel-measurable function.

11 Theorem (Luzin? cf. [7]) Let $A \subseteq \sigma \times x$ be analytic with each fiber $A_{t}$ countable. Then there exists a sequence $\left(f_{i}: i \in \omega\right)$ of Borel-measurable functions $f_{i}: \sigma \rightarrow \chi_{0}$ such that for all $t, A_{t} \subseteq\left\{f_{i}(t): i \in \omega\right\}$.

12 Corollary (to Harrington's proof of Silver's theorem) Let $E \subseteq \mathscr{\sigma} \times x_{6}^{2}$ be co-analytic with each fiber $E_{t}$ an equivalence relation on $x_{\text {. }}$.

(a) The set $C=\left\{t \in \mathcal{J}: E_{t}\right.$ has only countably many equivalence classes $\}$ is co-analytic.

(b) There exists a sequence $\left(f_{i}: i \in \omega\right)$ of analytically measurable functions $f_{i}: \sigma \rightarrow \mathscr{\sigma}$ such that for all $t \in C,\left\{f_{i}(t): i \in \omega\right\}$ contains at least one representative of each $E_{t}$-equivalence class.

Proof: (b) Assume $\sigma=\chi_{0}=\eta(=\omega \omega)$ and for simplicity take $E$ to be $\Pi_{1}^{1}$ without parameters. Apply Theorem 9 to each $\Sigma_{1}^{1}$ subset of $\eta^{2}$, and extend the resulting uniformizations to analytically measurable functions $f_{i}$ defined on all of $\eta$. To see that the $f_{i}$ do what they should, invoke the following fact established in Harrington's proof: If an equivalence relation which is $\Pi_{1}^{1}$ in parameter $t$ has only countably many classes (as is the case for $E_{t}$ when $t \epsilon C$ ), then for every $x$ there is a set containing $x$ and contained in the equivalence class of $x$ which is $\Delta_{1}^{1}$ in parameter $t$.

(a) It follows from (b) that $C$ is at worst a $C$-set, being the inverse image under 
a $C$-measurable function of the co-analytic set:

$$
\left\{(t, \xi): \forall x \exists i x E_{t}(\xi)_{i}\right\}
$$

That $C$ itself is actually co-analytic follows from the detailed statement of Silver's theorem (according to which when a co-analytic equivalence has uncountably many classes there exist a perfect set $P$ and a continuous function $W$ such that for distinct $x$ and $y$ in $P, W(x, y)$ "witnesses" the inequivalence of $x$ and $y$ ). We do not enter into details, as (a) will not be needed below.

\section{Corollary Let $A \subseteq \sigma \times \mathscr{J} \times$ be analytic. Then:}

(a) (Luzin [7]) The set $C=\left\{t \in \sigma: A_{t}\right.$ is countable $\}$ is co-analytic.

(b) There exists a sequence $\left(f_{i}: i \in \omega\right)$ of analytically measurable functions $f_{i}: \mathcal{F} \rightarrow \mathscr{X}_{0}$ such that for all $t \in C, A_{t} \subseteq\left\{f_{i}(t): i \in \omega\right\}$.

Proof: Apply Corollary 12 to $E=\left\{(t,(x, y)): x=y \vee\left(x \notin A_{t} \& y \notin A_{t}\right)\right\}$.

14 Alternative Proof: There are very few results in descriptive set theory that can be stated classically (as results of topology, not recursion theory) and proved absolutely (in $Z F C$, without extra set-theoretic hypotheses) whose known proofs require the use of logical methods (recursion theory, forcing, and so on). For instance, there are no such results in [9]. Silver's theorem (even with Harrington's proof) is one such result. From some points of view it is desirable to stick to classical methods of proof (among which we count gametheory) when possible. So let us outline a classical proof of Corollary 13.

Such a proof can be obtained by combining two things. On the one hand we have a game-theoretic analysis of cardinality due in its original version to M. Davis (answering a question of L. Dubins), and in the "unfolded" version used below to D. A. Martin (according to information supplied by the referee).

Let $A \subseteq C\left(=\omega_{2}\right)$ be analytic; write it as the projection of a closed $K \subseteq \subset \times \sim$. Consider the game $G^{*}(A)$ :

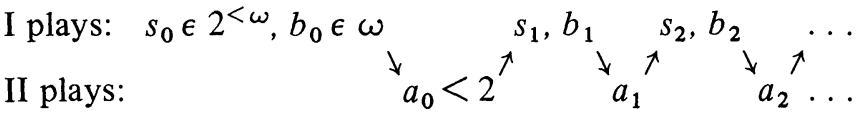

with I winning iff $(\alpha, \beta) \in K$, where:

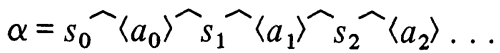

$$
\begin{aligned}
& \beta=\left\langle b_{0}, b_{1}, b_{2}, \ldots\right\rangle \text {. }
\end{aligned}
$$

(Note that the payoff set is closed.) Then (cf. [9], pp. 295-297): $A$ is countable iff II has a winning strategy in $G^{*}(A)$. The proof shows that from such a strategy a countable set including $A$ can be developed in a particularly simple fashion.

On the other hand, we have the work of Moschovakis on the complexity of winning strategies for simple games. Let $L \subseteq \eta^{2}$ be closed, and for each fiber $L_{t}$ consider the usual game $G\left(L_{t}\right)$. Then (cf. [9], Part 6E): The set $W_{\text {II }}$ of those $t$ for which II has a winning strategy in $G\left(L_{t}\right)$ is co-analytic. There is an analytically measurable function $S_{\mathrm{II}}$ assigning a suitable strategy to each $t$ in

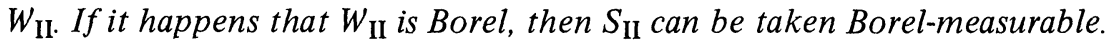


It should be clear how a proof of Corollary 13 (and of Theorem 11, for that matter) can be constructed from these materials.

15 Alternative Proof: Taking $\sigma=\varnothing=q$ and assuming (a) of Corollary 13, we can produce the functions required by (b) of Corollary 13 given any suitably measurable function $Q$ uniformizing the co-analytic set:

$$
P=\left\{(t, \xi): t \in C \& \forall x\left(x \in A_{t} \rightarrow \exists i x=(\xi)_{i}\right)\right\} .
$$

Now by M. Kondo's theorem ([9], Part 4E) $P$ can be uniformized by a function $Q$ which has co-analytic graph and hence is $\Delta_{2}^{1}$-measurable. In $Z F C$, we cannot prove that all $\Delta_{2}^{1}$ sets are absolutely measurable, but this does follow from any of the following extra set-theoretic hypotheses:

$M A$ Martin's Axiom plus the negation of the Continuum Hypothesis

$M C \quad$ The existence of a measurable (or other large enough) cardinal

$P D$ (Enough of) Projective Determinateness.

So we can get a cheap proof of Corollary 13 (with "analytically" weakened to "absolutely") from famous theorems if we are willing to go a little beyond $Z F C$. This illustrates what B. Russell called "the advantages of theft over honest toil".

Actually, a little metamathematical bookkeeping turns this dishonest proof into an honest one. R. M. Solovay, and independently Fenstad and Normann [4] have introduced the notion of an absolutely $\Delta_{2}^{1}$ set and shown (using absoluteness considerations and random-real forcing) that all such sets are absolutely measurable. And the function $Q$ above can be taken to be absolutely $\Delta_{2}^{1}$-measurable if one is careful.

We have now assembled all the uniformization theorems needed below.

16 Definitions Let $\Lambda$ be a so-called point-class, e.g., the Borel sets or the $C$-sets. Let $Y \subseteq \mathcal{\sigma} \times \%$ belong to $\Lambda$, with every fiber $Y_{t} \neq \phi$. A $\Lambda$-parametrized family of preference orders on $Y$ is an $R$ satisfying:

$R \subseteq\left\{(t,(x, y)): x, y \in Y_{t}\right\}$

$R$ belongs to $\Lambda$.

For all $t \in \mathscr{F}, R_{t}$ is a closed preference order on $Y_{t}$ (closure being understood relative to the subspace topology on $Y_{t}$ ).

We often write $\leqslant_{t}$ for $R_{t}$ and use the self-explanatory notation $<_{t}, \equiv_{t},[]_{t}$.

A $\Lambda$-parametrized family of utility functions on $Y$ is an $F$ satisfying:

$F: Y \rightarrow[0,1]$

$F$ is $\Lambda$-measurable.

For all $t \in \tilde{J}, F_{t}(y)=F(t, y)$ defines a continuous utility function on $Y_{t}$ (continuity being understood relative to the subspace topology on $\left.Y_{t}\right)$.

Finally, F represents $R$ if for all $t \in \tilde{J}, F_{t}$ represents $R_{t}$.

In [8] the question is taken up of when a Borel-parameterized family of preference orders can be expected to admit a representation by a Borel-parameterized family of utility functions. The following general result has apparently been obtained independently by Mauldin and by Fremlin: 
17 Theorem Any Borel-parametrized family of preference orders can be represented by a $C$-parametrized (hence absolutely measurably parametrized) family of utility functions.

Proof: We carry out the proof of Theorem 5 "parameter-wise" invoking Theorem 9 at the place marked \#, and Corollary 12 at the place marked \#\#. Assume $\sigma=\mathscr{J}=q$, and fix a basis $\left(U_{i}: i \in \omega\right)$. Apply Theorem 9 to each $Y \cap\left(\mathscr{} \times U_{i}\right)$, and extend the resulting uniformizations to analytically measurable functions defined on all of $\chi$, and combine them to obtain an analytically measurable function $Z$ uniformizing the co-analytic set $D$ defined by:

$$
D(t, \zeta) \longleftrightarrow \forall i(\zeta)_{i} \in Y_{t} \& \forall i\left(Y_{t} \cap U_{i} \neq \phi \rightarrow(\zeta)_{i} \in U_{i}\right)
$$

Thus $\left((Z(t))_{i}: i \epsilon \omega\right)$ behaves like $\left(z_{i}: i \epsilon \omega\right)$ in the proof of Theorem 5 .

Referring back to that proof (and invoking the Supplement to Proposition 2) it is not hard to obtain a Borel-measurable $H: Y \times q \rightarrow[0,1]$ such that when $\zeta=Z(t)$, then the function $H_{t, \zeta}(x)=H((t, x), \zeta)$ is a continuous utility function on $Y_{t}$ satisfying the following:

$$
\begin{aligned}
& x \leqslant t y \rightarrow H_{t, \zeta}(x) \leqslant H_{t, \zeta}(y) \\
& x<_{t} y \& H_{t, \zeta}(x)=H_{t, \zeta}(y) \rightarrow[x]_{t} \text { is a jump class }
\end{aligned}
$$

i.e., $H_{t, \zeta}$ behaves like $h$ in the proof of Theorem 5 .

Define now an analytic $J$ and a co-analytic $E$ by:

$$
\begin{aligned}
& J(t, \zeta, x) \longleftrightarrow x \in Y_{t} \& \exists y \in Y_{t}\left(x<_{t} y \& \sim \exists i\left(x<_{t}(\zeta)_{i}<_{t} y\right)\right) \\
& E((t, \zeta),(x, y)) \longleftrightarrow x=y \vee\left\{D(t, \zeta) \&\left[x \equiv_{t} y \vee(\sim J(t, \zeta, x) \& \sim J(t, \zeta, y))\right]\right\}
\end{aligned}
$$

Note that each $E_{t, \zeta}$ is an equivalence relation, and that when $\zeta=Z(t)$, then its equivalence classes are precisely the jump class of $\leqslant_{t}$ plus one extra "waste" class. Apply Corollary 12 to $E$, and extend the resulting functions to analytically measurable functions defined on all of $n$, combine them, and compose with $Z$ to obtain a $C$-measurable function $\Xi: q \rightarrow \eta$ such that for all

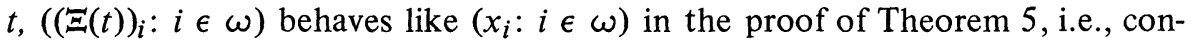
tains at least one representative of each jump class of $\leqslant_{t}$.

Next, suitably composing $Z, \Xi$, and the characteristic function of $J$, obtain a $C$-measurable function $M: \bar{\phi} \rightarrow C$ such that for all $t, M(t)$ behaves like $\left(m_{i}: i \in \omega\right)$ in the proof of Theorem 5 , i.e., $M(t)(i)=1$ iff $(\Xi(t))_{i}$ is a jump class of $\leqslant_{t}$.

Finally, define Borel-measurable functions as follows:

$$
\begin{aligned}
G_{i}((t, x), \xi) & =\left\{\begin{array}{l}
1 \text { if }(\xi)_{i} \epsilon Y_{t} \&(\xi)_{i}<_{t} x \\
0 \text { otherwise }
\end{array}\right. \\
G^{\prime}((t, x),(\xi, \mu)) & =\frac{1}{2} \sum_{i} 2^{-i} \mu(i) G_{i}((t, x), \xi) \\
G((t, x),(\zeta, \xi, \mu)) & =\frac{1}{2} H((t, x), \zeta)+\frac{1}{2} G^{\prime}((t, x),(\xi, \mu)) .
\end{aligned}
$$

Comparison with the definitions of $f_{i}, f^{\prime}, f$ in the proof of Theorem 5 shows that to obtain our desired representation, it will suffice to set:

$$
F(t, x)=G((t, x),(Z(t), \Xi(t), M(t))) .
$$


18 Alternative Proof: To avoid direct use of Corollary 12, carry out the Alternative Proof of Theorem 5 "parameter-wise", invoking Theorem 9 at the places marked \#, and Corollary 13 at the places marked \#\#\#.

19 Corollary (Wesley [13]) Let $R$ be a Borel-parametrized family of preference orders, and $\nu$ a $\sigma$-finite Borel measure on $\sigma$. Then there exist a Borel $N$ with $\nu N=0$, and a Borel-parametrized family of utility functions $F$ representing the restriction of $R$ to $\sigma-N$.

Proof: Take $N$ so that the restrictions to $\sigma-N$ of $Z, \Xi$, and $M$ in the proof of Theorem 17 are Borel-measurable.

20 Theorem (cf. [8], Theorem 5.6) Let $Y \subseteq \sigma \times \mathscr{J}_{6}$ be Borel with each fiber $Y_{t} \sigma$-compact. Then any Borel-parametrized family of preference orders on $Y$ can be represented by a Borel-parametrized family of utility functions.

Proof: Follow the Alternative Proof of Theorem 17, but invoking Theorem 10 in place of Theorem 9, and Theorem 11 in place of Corollary 13. We omit the details, which include some uses of part (a) of Theorem 10 to verify that certain sets are Borel, because this result improves only infinitesimally on what is proved by Mauldin.

21 Historical Note: Wesley [13] obtained Corollary 19 using absoluteness considerations and random-real forcing. A proof of Theorem 17 along similar lines can be obtained by combining our Remarks 15 and 18 above. Mauldin [8] obtained a purely classical proof of Corollary 19. A classical proof of Theorem 17 can be obtained by combining our Remarks 14 and 18. Mauldin [8] also gave a proof of Theorem 17 with " $C$-measurable" weakened to "absolutely measurable", assuming $M A$. Again this invites comparison without Remarks 15 and 18. It is hoped that the frequency with which [8] has been cited makes clear the extent of our indebtedness to Mauldin.

22 Methodological Remark: One often meets with uses of uniformization or selection theorems in problems on the (hyper-)theoretical fringes of mathematical disciplines normally thought of as applied (probability, control theory, etc.). If one's interest is genuinely in applications-presumably in constructing highly idealized models of empirical phenomena-then there can be no legitimate objection to using hypotheses like $P D$ going beyond the usual $Z F C$ axioms of set theory, at least so long as one can be reasonably confident that they are consistent: For a model inside a model of set theory is still a model.

But if one is willing to assume enough determinateness, then uniformization problems can be made to disappear once and for all, and it will never be necessary again to prove special selection theorems for special applications (as we had to prove Corollary 12 or Corollary 13 to get Theorem 17). For in applications it will never be necessary to go beyond the family 24 of so-called hyperprojective sets, which has the following properties (the first due to Mycielski and Swierczkowski, the rest to Moschovakis):

all sets in are absolutely measurable;

24 contains the open sets and is stable under complementation, countable union, and projection; and any set in 24 can be uniformized by an 24 -measurable function. 
Thus working inside 2 it is almost true that one can use the Axiom of Choice freely, and still assume that all the functions one gets are measurable.

\section{REFERENCES}

[1] Arsenin, V. Y., "Sur les projections de certains ensembles B," Comptes Rendus de l'Académie des Science de l'U.R.S.S., vol. 27 (1940), pp. 107-109.

[2] Choban, M., “On B-measurable selections," Soviet Mathematics Doklady, vol. 13 (1972), translation, pp. 1473-1477.

[3] Debreu, G., "Continuity properties of Paretan utility," International Economic Review, vol. 5 (1964), pp. 285-293.

[4] Fenstad, J. E. and D. Normann, "On absolutely measurable sets," Fundamenta Mathematicae, vol. 81 (1974), pp. 91-98.

[5] Kechris, A. S., D. A. Martin, and Y. N. Moschovakis, editors, Cabal Seminar 77-79, Springer Lecture Notes in Mathematics \#839, Springer, Berlin-Heidelberg-New York, 1980.

[6] Krantz, D. H., R. D. Luce, P. Suppes, and A. Tversky, Foundations of Measurement, Academic Press, New York, 1971.

[7] Luzin, N., Leçons sur les Ensembles Analytiques et leurs Applications, 2nd Ed., Chelsea, New York, 1972.

[8] Mauldin, R. D., "Measurable constructions of preference orders," Transactions of the American Mathematical Society, to appear.

[9] Moschovakis, Y. N., Descriptive Set Theory, North Holland, Amsterdam, 1980.

[10] von Neumann, J., "On rings of operators: Reduction theory," Annals of Mathematics, vol. 30 (1949), pp. 401-485.

[11] Wagner, D. H., "Survey of measurable selection theorems," SIAM Journal of Control and Optimization, vol. 5 (1977), pp. 859-903.

[12] Wagner, D. H., "Survey of measurable selection theorems: An update," in Measure Theory: Oberwolfach 1979, ed., D. Közlow, Springer Lecture Notes in Mathematics \#794, Springer, Berlin-Heidelberg-New York, 1980.

[13] Wesley, E., "Borel preference orders in markets with a continuum of traders," Journal of Mathematical Economics, vol. 3 (1976), pp. 155-165.

[14] Yankov, V., "Sur l'uniformisation des ensembles A," Comptes Rendus de l'Académie des Sciences de l'U.R.S.S., vol. 30 (1941), pp. 597-598.

Department of Philosophy

Princeton University

Princeton, New Jersey 08544 